\title{
Array Antennas Based Joint Beamforming for IEEE 802.11n Wi-Fi
}

\author{
Cheng Guo, Liqiang Zhao \\ State Key Laboratory of Integrated Service Networks, \\ Xidian University, \\ Xi'an, Shaanxi 710071, China \\ guoch@stu.xidian.edu.cn
}

\author{
Whai-En Chen \\ Dept. of Computer Science \& Information Engineering \\ National Ilan University \\ Taiwan \\ wechen@niu.edu.tw
}

\begin{abstract}
In order to achieve array gain and spatial diversity or multiplexing gain simultaneously, a novel joint beamforming based on MIMO and array antenna techniques, referred to as J$B F$, is proposed for the LTE and Wifi downlink. Array gain is achieved from array antenna based beamforming, referred to as AA-BF. Spatial diversity and multiplexing gains are achieved from MIMO based beamforming, referred to as MIMO-BF. To implement J-BF, i.e., joint AA-BF and MIMO-BF, an access point (AP) is equipped with separate array antennas. Before sending any data-frame in the J-BF mode, firstly, based on the estimated omni-directional CSI, the directional beam can be formed by the array antenna, and the array gain is achieved. Secondly, based on the estimated directional CSI, MIMO-BF is implemented to achieve the spatial diversity or multiplexing gain. More importantly, the J-BF algorithm maintains compatibility with 802.11n and there is not any change in terminals. Simulation results show that the proposed scheme can support the joint AA$B F$ and MIMO-BF effectively and provide much higher array gain or spatial gains than the traditional MIMO or array antenna respectively.
\end{abstract}

Keywords: Beamforming; Array Antenna; MIMO

\section{INTRODUCTION}

Due to the demand for higher date rates in wireless communicaitons, some key technologies are proposed to enhance the capacity of wireless networks, e.g., LTE/LTE-A and Wi-Fi. MIMO precoding [1] is a very promising technology in LTE and Wifi, which is a multi-stream beamforming (BF) technique and can significantly improve system capacity and diversity gain, referred to as MIMO-BF [2]. MIMO-BF has demonstrated significant capacity improvement in scatter-rich wireless environments, e.g., the Rayleigh independent identically distributed (i.i.d) channel. In order to exploit the diversity or capacity gain in MIMO systems as large as possible, proper transmission precoding has been proved to be very effective under the condition that the channel knowledge information is available at the transmitter side[3]. Nevertheless, if the scattering is not rich enough at all, line-ofsight (LOS) component will be presented with a high probability, which can bring bad influence on MIMO-BF. As we all know, the LOS is a component that exists by virtue of a direct path between the transmitter and the receiver. And the LOS component manifests itself if the separation distance between antennas is less than the coherent distance of the channel.

In the traditional array antenna based beamfroming, referred to as AA-BF [4], the same signal is emitted from each of the transmit antenna arrays with appropriate phase and amplitude so that the signal power is maximized at the receiver. AA-BF can enhance signal quality and increase system capacities without increasing the total transmit power [5]. Compared with MIMO-BF, AA-BF requires the LOS component, and the antenna arrays are closely co-located.

Hence, there is an urgent demand for joint beamforming, referred to as J-BF, to take full advantage of both AA-BF and MIMO-BF simultaneously in variable environments. In this paper, we present a J-BF strategy, i.e., joint MIMO-BF and AA-BF, for the LTE or Wifi downlink, which can provide spatial diversity, multiplexing gain and array gain simultaneously in different wireless environments. Moreover, the J-BF is implemented in the transmitter, i.e., access point (AP), and there is not any change in the receiver, i.e., mobile station (STA). The proposed J-BF is effective in both LTE/LTE-A and Wi-Fi, and for simplicity, we only consider implementing it in Wi-Fi in this paper.

The paper is organized as follows. The system model is introduced in Section II. The joint MIMO-BF and AA-BF algorithm is proposed in Section III. Simulation results are provided in Section IV. In Section V, the conclusion is given.

\section{SYSTEM MODEL FOR JOINT BEAMFORMING}

\section{A. System Model}

To take full advantage of both MIMO-BF and AA-BF simultaneously, each AP is equipped with $N_{T}$ array antennas, each of which consists of $N_{a}$ arrays, as shown in Fig. 1. These array antennas are well separated from each other by a distance $D_{c}$ which exceeds the coherent distance of the channel. Moreover, in order to achieve the array gain as large as possible, the arrays within each antenna are highly close to each other [6]. According to the standard of Wifi, all STAs are equipped with $N_{R}$ omni-directional antennas. 
Consider a signal vector $\mathbf{s}$ of dimensional $N_{S S} \times 1$ where $N_{S S}$ is the number of data streams. The signal vector is multiplied by a matrix for MIMO-BF, $\mathbf{W} \in C^{N_{T} \times N_{S S}}$, and a matrix for AA-BF, $\mathbf{V} \in C^{\left(N_{T} \times N_{a}\right) \times N_{T}}$, prior to transmission. So at the receiver, the received signal vector $\mathbf{r}$ of dimensional $N_{R} \times 1$ is multiplied by the post-processing matrix $\mathbf{P}$ in order to extract the desired signal from the receiver perfectly, which can be expressed as:

$$
\widetilde{\mathbf{s}}=\mathbf{P r}=\mathbf{P}(\mathbf{H V W s}+\mathbf{n})
$$

where the channel matrix $\mathbf{H} \in C^{N_{R} \times\left(N_{T} \times N_{a}\right)}$, and the channel noise $\mathbf{n} \in C^{N_{R} \times 1}$, which is drawn from an ensemble of i.i.d complex Gaussian random variables with zero mean and variance $\sigma_{n}^{2}$.

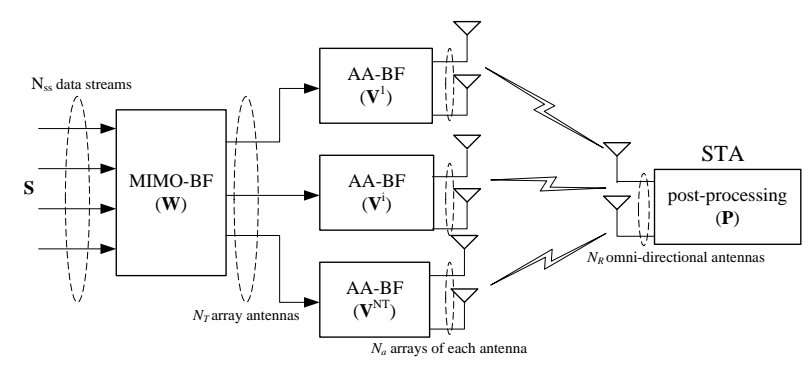

Fig. 1 System model for joint beamforming

The AA-BF matrix $\mathbf{V}$ could be expressed as: $\mathbf{V}=\operatorname{diag}\left\{\mathbf{v}_{N_{a} \times 1}^{1}, \mathbf{v}_{N_{a} \times 1}^{2}, \ldots, \mathbf{v}_{N_{a} \times 1}^{N_{T}}\right\}$, where $\mathbf{v}_{N_{a} \times 1}^{i}$ is the AA-BF vector of the $i$-th antenna at the transmitter. In order to constrain the total transmit energy, the matrix satisfies $\left(\mathbf{v}_{N_{a} \times 1}^{i}\right)^{H} \mathbf{v}_{N_{a} \times 1}^{i}=1$, where ()$^{H}$ denotes the conjugate transpose operator.

The channel matrix $\mathbf{H}$ is expressed as: $\mathbf{H}=\left[\mathbf{H}_{N_{R} \times N_{a}}^{1}, \mathbf{H}_{N_{R} \times N_{a}}^{2}, \ldots, \mathbf{H}_{N_{R} \times N_{a}}^{N_{T}}\right]$, where $\mathbf{H}_{N_{R} \times N_{a}}^{i}$ is the channel matrix from the $i$-th antenna with $N_{a}$ arrays at the transmitter to the receiver. For simple analysis, a set of channel models in Wifi [7], is given in Tab. 1.

TAB. 1 CHANNEL MODELS

\begin{tabular}{|c|c|c|c|}
\hline \multicolumn{2}{|c|}{ RMS delay } & \multirow{2}{*}{ Environment } & \multirow{2}{*}{ Example } \\
\hline Mod & preac & & \\
\hline $\mathrm{A}$ & 0 & N/A & N/A \\
\hline B & 15 & Residential & Intra-room, room to room \\
\hline $\mathrm{C}$ & 30 & Residential/small office & Conference room, classroom \\
\hline $\mathrm{D}$ & 50 & Typical office & large conference room \\
\hline $\mathrm{E}$ & 100 & Large office & Multi-story office \\
\hline $\mathrm{F}$ & 150 & Large space & city square \\
\hline
\end{tabular}

\section{JOINT BEAMFORMING ALGORITHM}

At the beginning, the transmitter works in the omnidirectional mode as it does not know both the MIMO-BF and
AA-BF matrix (i.e., $\mathbf{W}$ and $\mathbf{V}$ ) corresponding to its receivers. Before calculating the two vital matrixes in the J-BF mode, the transmitter has to get the relevant CSI by which the $\mathbf{W}$ and $\mathbf{V}$ can be naturally obtained. The reciprocity between the uplink and downlink channels can be exploited to achieve the CSI [8]. Please note that the required CSI for MIMO-BF and AA-BF is tremendous different.

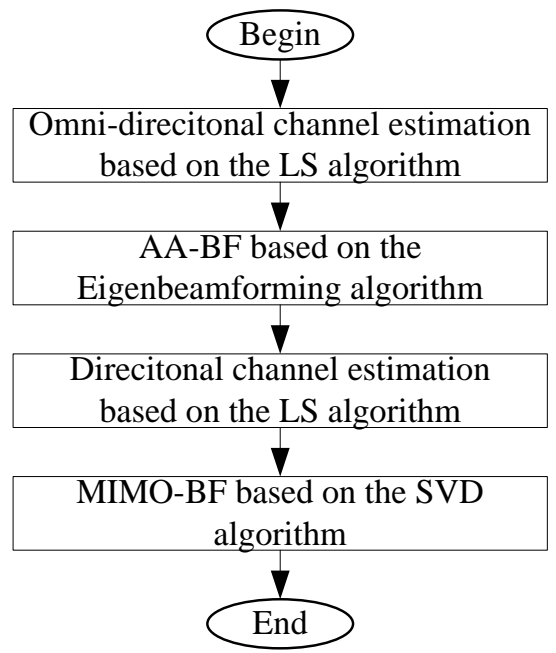

Fig. 2 Flow chart for J-BF

To implement J-BF in the LTE downlink, as shown in Fig. 2, firstly, the AP estimates the CSI of the omni-directional channel through the uplink, and calculates the AA-BF matrix $\mathbf{V}$ based on the estimated omni-directional CSI. Since then, the AP transmits its packets in the AA-BF mode. Secondly, after implementing AA-BF, the AP estimates the CSI of the AA-BF channel through the uplink, and calculates the MIMO-BF matrix $\mathbf{W}$ based on the estimated directional CSI. Finally, the J$\mathrm{BF}$ is implemented and the benefits including diversity, array and multiplexing gains can be obtained through the J-BF mode eventually. The detailed steps are shown in the following:

- Omni-directional channel estimation

Wi-Fi is a typical OFDM-based packet transmission system, which divides the bandwidth into $N$ orthogonal subcarriers. And the training symbols for $N$ subcarriers can be obviously represented by the following diagonal matrix:

$$
\mathbf{X}=\left[\begin{array}{ccc}
X[0] & \cdots & 0 \\
\vdots & \ddots & \vdots \\
0 & \cdots & X[N-1]
\end{array}\right]
$$

where $X[k]$ represents the training symbols at the $k$-th subcarrier, $k=0,1,2, \ldots, N-1$, with $E\{X[k]\}=0 \quad$ and $\operatorname{Var}\{X[k]\}=\sigma_{x}^{2}$.

Given that the channel gain from the STA to the i-th antenna at the AP is $H_{N_{a} \times N_{R}}^{i}[k]$ for the subcarrier $k$, the received training signal $\mathbf{Y}$ can be expressed as 
$\mathbf{Y} \triangleq\left[\begin{array}{c}Y[0] \\ Y[1] \\ \vdots \\ Y[N-1]\end{array}\right]$

$=\left[\begin{array}{ccc}X[0] & \cdots & 0 \\ \vdots & \ddots & \vdots \\ 0 & \cdots & X[N-1]\end{array}\right]\left[\begin{array}{c}H_{N_{a} \times N_{R}}^{i}[0] \\ H_{N_{a} \times N_{R}}^{i}[1] \\ \vdots \\ H_{N_{a} \times N_{R}}^{i}[N-1]\end{array}\right]+\left[\begin{array}{c}Z[0] \\ Z[1] \\ \vdots \\ Z[N-1]\end{array}\right]$

$=\mathbf{X H}_{N_{a} \times N_{R}}^{i}+\mathbf{Z}$

where the channel vector $\mathbf{H}_{N_{a} \times N_{R}}^{i}$ from the STA to the i-th antenna at the AP can be expressed as:

$\mathbf{H}_{N_{a} \times N_{R}}^{i}=\left[H_{N_{a} \times N_{R}}^{i}[0], H_{N_{a} \times N_{R}}^{i}[1], \ldots, H_{N_{a} \times N_{R}}^{i}[N-1]\right]^{T}$ and the noise vector $\mathbf{Z}=[Z[0], Z[1], \ldots, Z[N-1]]^{T}$ with $E\{\mathbf{Z}[k]\}=0$ and $\operatorname{Var}\{Z[k]\}=\sigma_{z}^{2}$.

In the following discussion, we assume that $\hat{\mathbf{H}}$ denotes the estimate values of the channel matrix $\mathbf{H}_{N_{a} \times N_{R}}^{i}$. And the leastsquare (LS) estimation algorithm is used to get $\hat{\mathbf{H}}$ in such a way that the following cost function is minimized:

$$
\begin{aligned}
J(\hat{\mathbf{H}}) & =\|\mathbf{Y}-\mathbf{X} \hat{\mathbf{H}}\|^{2} \\
& =(\mathbf{Y}-\mathbf{X} \hat{\mathbf{H}})^{H}(\mathbf{Y}-\mathbf{X} \hat{\mathbf{H}}) \\
& =\mathbf{Y}^{H} \mathbf{Y}-\mathbf{Y}^{H} \mathbf{X} \hat{\mathbf{H}}-\hat{\mathbf{H}}^{H} \mathbf{X}^{H} \mathbf{Y}+\hat{\mathbf{H}}^{H} \mathbf{X}^{H} \mathbf{X} \hat{\mathbf{H}}
\end{aligned}
$$

By setting the derivative of the function with respect to $\hat{\mathbf{H}}$ to zero, we can obtain

$$
\frac{\partial J(\hat{\mathbf{H}})}{\partial \hat{\mathbf{H}}}=-2\left(\mathbf{X}^{H} \mathbf{Y}\right)+2\left(\mathbf{X}^{H} \mathbf{X} \hat{\mathbf{H}}\right)^{*}=0
$$

Hence, $\mathbf{X}^{H} \mathbf{X} \hat{\mathbf{H}}=\mathbf{X}^{H} \mathbf{Y}$, which gives the solution to the LS estimation as

$$
\hat{\mathbf{H}}=\left(\mathbf{X}^{H} \mathbf{X}\right)^{-1} \mathbf{X}^{H} \mathbf{Y}=\mathbf{X}^{-1} \mathbf{Y}
$$

With respect to the reciprocity between the uplink and downlink channels, the downlink CSI from the i-th antenna at the AP to STA, $\mathbf{H}_{N_{R} \times N_{a}}^{i}$, can be represented as:

$$
\mathbf{H}_{N_{R} \times N_{a}}^{i}=(\hat{\mathbf{H}})^{T} .
$$

- Array antenna based beamforming

There have been several effective AA-BF algorithms, e.g., direction-of-arrival (DOA) based beamforming [9] and eigenbeamforming[10], for the AP to get the directional beams based on the estimated omni-directional CSI. Eigenbeamforming takes full advantage of the spatial correlations by transmitting over the strongest beam to a given user which can increase system capacity and reduce the interference from others, and we take it as the AA-BF algorithm in this paper.

Based on the estimated omni-directional CSI, the spatial correlation matrix between the $i$-th antenna of the AP and its STA is obtained as follows:

$$
\mathbf{C}^{i}=E\left\{\left(\mathbf{H}_{N_{R} \times N_{a}}^{i}\right)^{H} \mathbf{H}_{N_{R} \times N_{a}}^{i}\right\} \quad i=1,2, \ldots, N_{T}
$$

The sorted eigenvalue decomposition of the correlation matrix $\mathbf{C}^{i}$ in the descending order is expressed as:

$$
\mathbf{C}^{i}=\mathbf{D}^{i} \Lambda^{i}\left(\mathbf{D}^{i}\right)^{H}=\sum_{k=1}^{N_{a}} \lambda_{k, i} \mathbf{D}_{k, i}\left(\mathbf{D}_{k, i}\right)^{H} \quad i=1,2, \ldots, N_{T}
$$

where $\mathbf{D}_{1, i}$ corresponding to the largest eigenvalue $\lambda_{1, i}$, is the principal eigenvector of $\mathbf{C}^{i}$.

In eigenbeamforming, the AA-BF vector of the $i$-th transmitter antenna at the AP, $\mathbf{v}^{i}=\mathbf{D}_{1, i}$.

- Directional channel estimation

Obviously, after implementing AA-BF, the channel has been changed seriously, which is not the initial omnidirectional channel. So we have to estimate the CSI of the directional channel, referred to as $\mathbf{H}_{A A-B F}$, again. Similarly, it is easy to estimate the AA-BF channel based on the LS algorithm as shown in the first step.

- MIMO based beamforming

Several effective MIMO-BF algorithms have being studied, e.g., Singular Value Decomposition (SVD) [11], Geometric Mean Decomposition (GMD) [12], etc. In this paper, we just take SVD into consideration.

The SVD of $\mathbf{H}_{A A-B F}$ can be expressed as:

$$
\mathbf{H}_{A A-B F}=\mathbf{U} \boldsymbol{\Sigma} \mathbf{W}^{H}
$$

where $\mathbf{U}$ and $\mathbf{W}$ are unitary matrices; $\boldsymbol{\Sigma}$ is a diagonal matrix of the singular values of $\mathbf{H}_{A A-B F}$ in the descending order. The MIMO-BF matrix $\mathbf{W}$ is achieved now.

Up to now, the AP has got the AA-BF and MIMO-BF matrix, and then sends its packets in the J-BF mode.

- Decoding

In order to decode the desired signal from each antenna at the STA, the received signal $\mathbf{r}$ should be multiplied by a weight matrix $\mathbf{P}$ such that

$$
\widetilde{\mathbf{s}}=\mathbf{P r}
$$

Some popular decoding methods can be exploited to calculate $\mathbf{P}$, e.g., the zero-forcing ( $\mathrm{ZF}$ ) and minimum mean square error (MMSE) algorithm recommended. In order to maintain compatible with $802.11 \mathrm{n}$ without any change in STA, the $\mathrm{ZF}$ is taken into account.

The $\mathrm{ZF}$ technique nullifies the interference by the following matrix: 


$$
\mathbf{P}=\left((\mathbf{H V W})^{H} \mathbf{H V W}\right)^{-1}(\mathbf{H V W})^{H}
$$

where ()$^{H}$ denotes the Hermitian transpose operation. That is to say, it inverts the effect of channel as

$$
\widetilde{\mathbf{s}}=\mathbf{P r}=\mathbf{s}+\tilde{\mathbf{n}}
$$

where $\tilde{\mathbf{n}}=\mathbf{P n}=\left((\mathbf{H V W})^{H} \text { HVW }\right)^{-1}(\mathbf{H V W})^{H} \mathbf{n}$.

\section{Simulation Results}

In order to evaluate the proposed joint beamforming algorithm, the following simulations are performed under the indoor channel model D [13]. The values of the parameters used to obtain numerical results for simulations are specified in Wi-Fi, and some of the main parameters are given in Tab. 2. The distance between antennas at the AP is $0.5 \lambda$ and that between arrays in an antenna is $0.25 \lambda$. For comparison, both the $N_{T} \times N_{a} \times N_{R}$ J-BF system and $N_{T} \times N_{R}$ MIMO system are evaluated under the same scenarios. For simplicity, we assume that both the AP and STA have the perfect CSI.

TAB. 2 SimULATION PARAMETERS

\begin{tabular}{|c|c|}
\hline Parameter & Value \\
\hline Frequency & $2.4 \mathrm{GHz}$ \\
\hline Bandwidth & $20 \mathrm{MHz}$ \\
\hline Sptial data stream & $1,2,3,4$ \\
\hline OFDM Symbol Length & $4 \mathrm{us}$ \\
\hline CP Length & $0.8 \mathrm{us}$ \\
\hline FFT Size & 64 \\
\hline Modulation Scheme & 64QAM \\
\hline Channel model & $\mathrm{D}$ \\
\hline AA-BF algorithm & Eigenbeamforming \\
\hline MIMO-BF algorithm & SVD \\
\hline Decoding & ZF \\
\hline
\end{tabular}

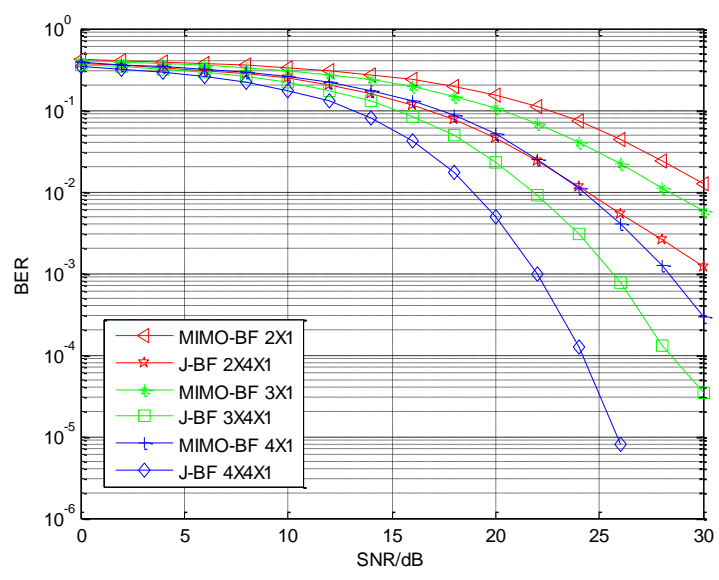

Fig. 3 BER performance of J-BF with variable $N_{T}$

The performance of $\mathrm{J}$-BF using variable number of antennas at the AP is examined in Fig. 3, where the number of independent data streams is fixed at one. Obviously, the BER performance of J-BF increases with the increasing transmit antennas as it provides a larger diversity order of $N_{T}$. Unfortunately, due to only one antenna at the receiver, we cannot get any spatial multiplex gain under this scenario. On the other hand, the performance of the $N_{T} \times N_{a} \times N_{R} \mathrm{~J}$-BF is much better than that of the $N_{T} \times N_{R}$ MIMO-BF as J-BF can obtain array gain to improve the link quality.

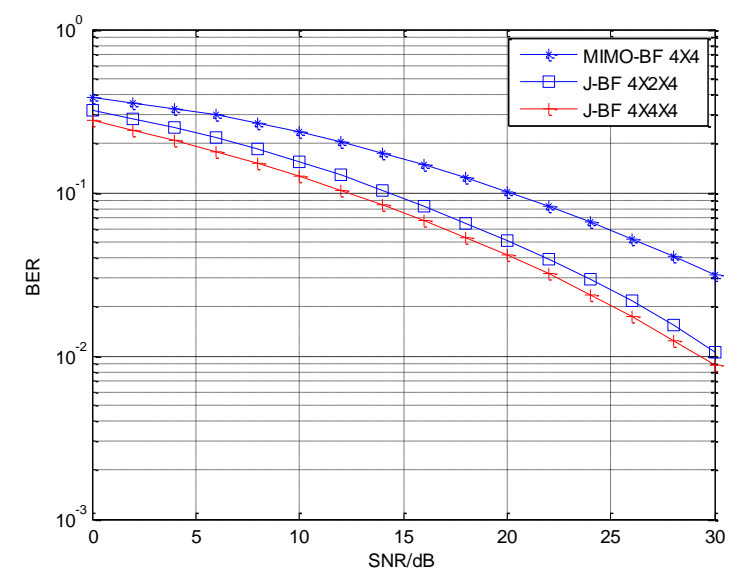

Fig. 4 BER performance of J-BF with variable $N_{a}$

Fig. 4 shows that the performance of $4 \times 2 \times 4$ and $4 \times 4 \times 4 \mathrm{~J}-\mathrm{BF}$ is much better than that of $4 \times 4$ MIMO-BF as J-BF can achieve array gains by means of AA-BF. Moreover, the $4 \times 2 \times 4 \mathrm{~J}-\mathrm{BF}$ scheme is $2 \mathrm{~dB}$ inferior to the $4 \mathrm{x} 4 \mathrm{x} 4 \mathrm{~J}-\mathrm{BF}$ scheme as the latter one can obtain a greater array gain with a larger number of arrays. Compared with Fig.3, the number of independent data streams is 4 as $N_{R}=4$ in Fig. 4 .

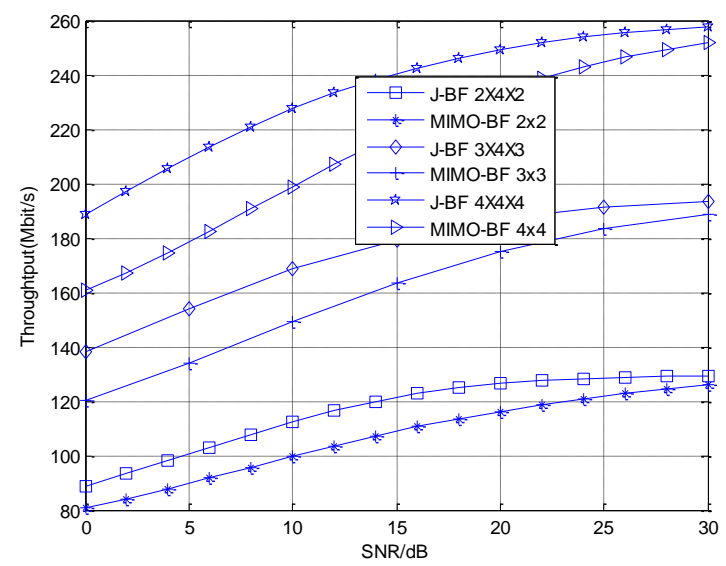

Fig. 5 Throughput of J-BF with variable $N_{R}$

Fig. 5 shows that system throughput of the $4 \times 4 \times 4 \mathrm{~J}-\mathrm{BF}$ scheme is much higher than that of the $3 \times 4 \times 3$ or $2 \times 4 \times 2 \mathrm{~J}-\mathrm{BF}$ scheme as it achieves a greater spatial multiplex gain and can support up to 4 independent spatial data streams while the latter two schemes only support 2 and 3 spatial data streams, respectively. Moreover, system throughput of the $4 \times 4 \times 4 \mathrm{~J}-\mathrm{BF}$ scheme is higher than that of the $4 \times 4$ MIMO-BF as J-BF can achieve array gains.

\section{CONCLUSIONS}

In this paper, in order to achieve both array gain and spatial diversity or multiplexing gain, a joint AA-BF and MIMO-BF, called J-BF, is presented. Firstly, the AP estimates the CSI of 
the omni-directional channel in the uplink, and calculates the AA-BF matrix based on the estimated omni-directional CSI. Since then, the AP transmits its packets in the AA-BF mode. Secondly, after implementing AA-BF, the AP estimates the CSI of the AA-BF channel in the uplink, and calculates the MIMO-BF matrix based on the estimated directional CSI. Hence, the J-BF algorithm is implemented, which is compatible with $802.11 \mathrm{n}$. Simulation results show that after taking full advantage of both array antennas and MIMO, J-BF can provide a much lower BER than the conventional AA-BF and MIMO-BF respectively.

Currently, we are carrying out the following two researches. Firstly, we are researching adaptive beam- forming algorithms based on joint AA-BF and MIMO-BF. Secondly, in order to introduce the joint beamforming, we are researching enhanced SDMA protocols for $802.11 \mathrm{n}$.

\section{ACKNOWLEDGMENT}

This work was supported in part by National Natural Science Foundation of China (No. 61372070), Natural Science Basic Research Plan in Shaanxi Province of China (2015JM6324), Hong Kong, Macao and Taiwan Science \& Technology Cooperation Program of China (2014DFT10320), EU FP7 Project MONICA (PIRSES-GA-2011-295222), and the 111 Project (B08038).

\section{REFERENCES}

[1] H. K. Jayesh, K. B. Huang, MIMO precoding enable spatial multiplexing,power allocation and adaptive modulation and coding[p],US 7,702,029 B2,Apr. 20,2010.

[2] Y. P. Wu, C. K. Wen, C. S. Xiao, X. Q. Gao, R. Schober, Linear MIMO precoding in jointly-correlated fading multiple access channels with finite alphabet signaling, IEEE International Conference on Communications(ICC), pp. 5306-5311, 2014.

[3] D. R. Qin, Z. Ding, S. Dasgupta, On Forward Channel Estimation for MIMO Precoding in Cooperative Relay Wireless Transmission Systems, IEEE transaction on Signal Processing, pp. 1265-1278, 2014.

[4] X. R. Wang, E. Aboutanios, M. Trinkle, M. G. Amin, Reconfigurable Adaptive Array Beamforming by Antenna Selection, IEEE Transactions on Singal Processing, pp. 2385-2396, 2014.

[5] J. Singh, S. Ramakrishna, On the feasibility of beamforming in millimeter wave communication systems with multiple antennaarrays, IEEE transactions on Wireless Communications, pp. 1, 2015.

[6] R. A. Soni, R.M. Buehrer and R. D. Benning,'Intelligent antenna system for cdma2000,"in IEEE Signal Processing Magazine, vol.19,no. 4,pp. $54-67,2002$.

[7] A. Stephens, B. Bjerke, etal.(2004).Usage Models,IEEE 802.1103/802r23.

[8] T. Luo, A. Damnjanovic, Malladi, channel estimation for wireless communication[p], US 2011/006653 A1, Feb. 3, 2001.

[9] D. J. Yeom, S. H. Park, etal. Performance analysis of beamspace MUSIC withbeamforming angle, International Conference on Singal Processing and Communication Systems(ICSPCS), pp. 1-5, 2014.

[10] J. Choi, On Coding and Beamforming for Large Antenna Arrays in mmWave Systems, IEEE Wireless Communications Letters, pp. 193-196, 2014

[11] H. Busche, A. Vanaev and H. Rohling, "SVD-based MIMO precoding and equalization schemes for realistic channel knowledge: Design criteria and performance evaluation," in Wireless Personal Communications,vol. 48,no. 3,pp. 347-359,2009.

[12] Y. Jiang, J. Li and W.W. Hager,"Joint transceiver design for MIMO communications using geometric mean decomposition," IEEE Trans. Signal Processing,vol. 53,no. 10,pp. 3791-3803,2005.

[13] V. Erceg, L. Schumacher, etal.(2004).TGn ChannelModels,IEEE 802.11-03/940r4 